\title{
An American Association for the Surgery of Trauma (AAST) prospective multi-center research protocol: outcomes of urethral realignment versus suprapubic cystostomy after pelvic fracture urethral injury
}

Rachel A. Moses ${ }^{1}$, John Patrick Selph ${ }^{2}$, Bryan B. Voelzke ${ }^{3}$, Joshua Piotrowski ${ }^{4}$, Jairam R. Eswara ${ }^{5}$, Bradley A. Erickson ${ }^{6}$, Shubham Gupta ${ }^{7}$, Roger R. Dmochowski ${ }^{8}$, Niels V. Johnsen ${ }^{8}$, Anand Shridharani ${ }^{9}$, Sarah D. Blaschko $^{10}$, Sean P. Elliott ${ }^{11}$, Ian Schwartz ${ }^{11}$, Catherine R. Harris ${ }^{12}$, Kristy Borawski ${ }^{13}$, Bradley D. Figler ${ }^{13}$, E. Charles Osterberg III ${ }^{14}$, Frank N. Burks ${ }^{15}$, William Bihrle III ${ }^{16}$, Brandi Miller ${ }^{17}$, Richard A. Santucci ${ }^{17}$, Benjamin N. Breyer ${ }^{18}$, Brian Flynn ${ }^{19}$, Ty Higuchi ${ }^{19}$, Fernando J. Kim ${ }^{19}$, Joshua A. Broghammer ${ }^{20}$, Angela P. Presson ${ }^{1}$, Jeremy B. Myers ${ }^{1}$; from the Trauma and Urologic Reconstruction Network of Surgeons (TURNS)

${ }^{1}$ Division of Urology, Department of Surgery, University of Utah, Salt Lake City, UT, USA; ${ }^{2}$ Department of Urology, University of Alabama at Birmingham, Birmingham, AL, USA; ${ }^{3}$ Department of Urology, Harborview Medical Center, University of Washington, Seattle, WA, USA; ${ }^{4}$ Department of Urology, Medical College of Wisconsin, Milwaukee, WI, USA; ${ }^{5}$ Division of Urology, Department of Surgery, Brigham and Women's Hospital, Boston, MA, USA; ${ }^{6}$ Department of Urology, University of Iowa, Iowa City, IA, USA; ${ }^{7}$ Department of Urology, University of Kentucky, Lexington, KY, USA; ${ }^{8}$ Department of Urology, Vanderbilt University Medical Center, Nashville, TN, USA; ${ }^{9}$ Department of Urology, University of Tennessee College of Medicine, Chattanooga, TN, USA; ${ }^{10}$ Division of Urology, Alameda Health System, Oakland, CA, USA; ${ }^{11}$ Division of Urology, Department of Surgery, Hennepin County Medical Center, University of Minnesota, Minneapolis, MN, USA; ${ }^{12}$ Santa Clara Valley Medical Center, San Jose, CA, USA; ${ }^{13}$ Department of Urology, University of North Carolina-Chapel Hill, Chapel Hill, NC, USA; ${ }^{14}$ Department of Surgery, University of Texas, Dell Medical School, Austin, TX, USA; ${ }^{15}$ Department of Urology, Oakland University William Beaumont School of Medicine, Royal Oak, MI, USA; ${ }^{16}$ Dartmouth Hitchcock Medical Center, Lebanon, NH, USA; ${ }^{17}$ Department of Urology, Detroit Medical Center, Detroit, MI, USA; ${ }^{18}$ Department of Urology, University of California-San Francisco, San Francisco, CA, USA; ${ }^{19}$ Department of Surgery (Urology), University of Colorado School of Medicine, Aurora, CO, USA; ${ }^{20}$ Department of Urology, University of Kansas Medical Center, Kansas City, Kansas, USA

Contributions: (I) Conception and design: JB Myers, RA Moses; (II) Administrative support: RA Moses; (III) Provision of study materials or patients: All authors; (IV) Collection and assembly of data: All authors; (V) Data analysis and interpretation: JB Myers, RA Moses; (VI) Manuscript writing: All authors; (VII) Final approval of manuscript: All authors.

Correspondence to: Rachel A. Moses, MD, MPH. Division of Urology, Department of Surgery, University of Utah, Salt Lake City, UT 84132, USA. Email: rachel.moses@hsc.utah.edu.

Background: Pelvic fracture urethral injuries (PFUI) occur in up to $10 \%$ of pelvic fractures. It remains controversial whether initial primary urethral realignment (PR) after PFUI decreases the incidence of urethral obstruction and the need for subsequent urethral procedures. We present methodology for a prospective cohort study analyzing the outcomes of PR versus suprapubic cystostomy tube (SPT) after PFUI. Methods: A prospective cohort trial was designed to compare outcomes between PR (group 1) and SPT placement (group 2). Centers are assigned to a group upon entry into the study. All patients will undergo retrograde attempted catheter placement; if this fails a cystoscopy exam is done to confirm a complete urethral disruption and attempt at gentle retrograde catheter placement. If catheter placement fails, group 1 will undergo urethral realignment and group 2 will undergo SPT. The primary outcome measure will be the rate of urethral obstruction preventing atraumatic passage of a flexible cystoscope. Secondary outcome measures include: subsequent urethral interventions, post-injury complications, urethroplasty complexity, erectile dysfunction (ED) and urinary incontinence rates.

Results: Prior studies demonstrate PR is associated with a $15 \%$ to $50 \%$ reduction in urethral obstruction. Ninety-six men (48 per treatment group) are required to detect a $15 \%$ treatment effect (80\% power, 0.05 
significance level, $20 \%$ loss to follow up/death rate). Busy trauma centers treat complete PFUI approximately 1-6 times per year, thus our goal is to recruit 25 trauma centers and enroll patients for 3 years with a goal of 100 or more total patients with complete urethral disruption.

Conclusions: The proposed prospective multi-institutional cohort study should determine the utility of acute urethral realignment after PFUI.

Keywords: Genitourinary trauma; pelvic fracture urethral injury (PFUT); primary realignment; urethral injury; urethroplasty

Submitted Aug 07, 2017. Accepted for publication Oct 23, 2017.

doi: $10.21037 /$ tau.2017.11.07

View this article at: http://dx.doi.org/10.21037/tau.2017.11.07

\section{Introduction}

Pelvic fracture urethral injury (PFUI) is a rare, potentially devastating genitourinary trauma, which occurs in up to $10 \%$ of pelvic fractures $(1,2)$. The injury occurs secondary to pelvic fracture sheering forces that tear the bulbomembraneous urethra in the area of the prostate apex or external sphincter (3). A well-described surgical procedure to treat this injury is to perform a posterior urethroplasty via a perineal approach, which has an $85 \%$ to $97 \%$ success rate $(4,5)$. However, this is usually delayed for several months to allow urethral inflammation to resolve (3) leading to a potentially cumbersome proximal urinary diversion with a suprapubic cystostomy tube (SPT) (6-8).

An alternative to SPT placement alone for the management of acute PFUI is immediate primary urethral realignment $(\mathrm{PR})$. Originally $\mathrm{PR}$ was described via an open approach with the passage of sounds or catheters through the pelvic hematoma and urethral defect $(9,10)$. More recently, PR has been accomplished endoscopically with the use of a simultaneous antegrade and retrograde cystoscope, which is used to bridge the urethral injury gap (11). Proponents of PR report up to $50 \%$ of patients undergoing this technique may avoid prolonged SPT, stricture, and urethroplasty altogether (10-12). Further, in bringing the severed ends of the urethra in closer proximity, PR may reduce the complexity of future urethroplasty if ultimately required (12).

There are several problems in the existing literature addressing acute management of PFUI with PR. Most studies are small, retrospective series reporting disparate efficacy of urethral realignment (11-14). Bias is potentially introduced into study results, as PR may be easier to perform in patients with minor disruptions or partial urethral injuries, which may heal regardless if $\mathrm{PR}$ is performed. This selection bias may make it appear the PR has a greater success than in reality. The current evidence for PFUI management is grade 3A (systematic review of case-control studies) in support of PR (15). Yet despite a grade 3A recommendation, studies have demonstrated that PR can worsen injuries (16), expose the patient to additional operations during trauma management (4). PR has been associated with higher risk of sexual dysfunction and incontinence $(11,16)$.

The controversy over the benefits of PR continues to be highly debated among urologists involved in trauma management; however, these arguments are generally backed with poor quality evidence and opinion. Therefore, we designed a prospective cohort multi-institutional study comparing $\mathrm{PR}$ in patients with complete urethral disruption versus SPT placement. We hypothesize that this study will determine the utility of PR compared to SPT for the acute management of PFUI.

\section{Methods}

\section{Study design}

A prospective observational cohort trial was designed to analyze outcomes between two PFUI management protocols. After receiving individual medical center institutional review board approval, participating centers will begin enrolling patients after PFUI. A centralized deidentified database via Research Electronic Data Capture (REDCap) will be used for data entry. The study has been advertised through the American Association for Surgery of Trauma Multi-Institutional Trials Committee (http:// www.aast.org/Research/MultiInstitutionalStudies.aspx). The inclusion criteria for this study includes: age $>18$ years old, male gender, blunt mechanism, and the presence of a traumatic pelvic fracture. Exclusion criteria will include 


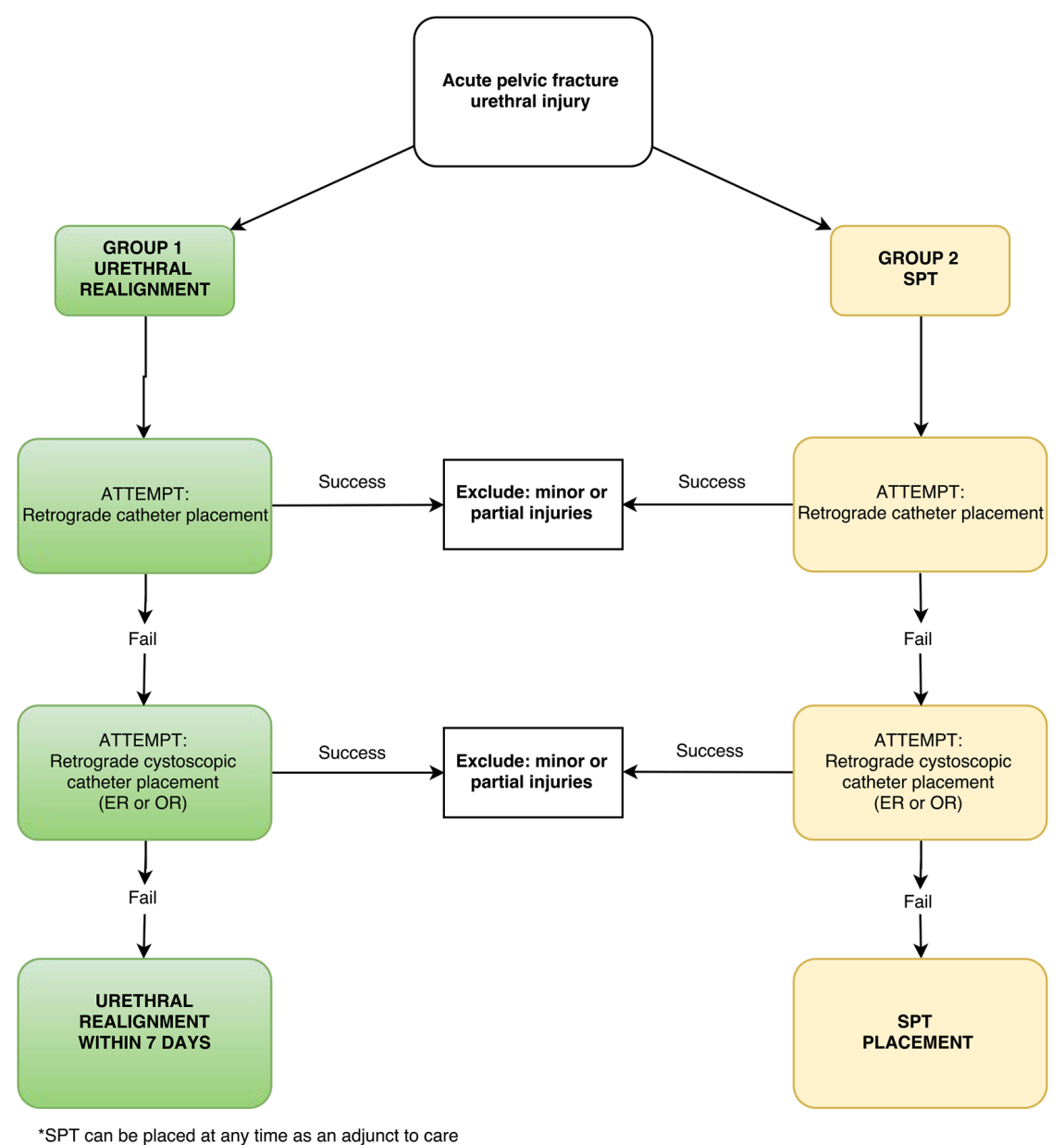

Figure 1 Summary of treatment groups. SPT, suprapubic cystostomy tube; ER, emergency room; OR, operating room.

patients treated outside of the agreed treatment protocol at a given center. We will also exclude patients that did not undergo cystoscopy to confirm a full transection prior to undergoing a SPT placement or PR. Other exclusion criteria include minor or partial injuries, death, inability to remove a Foley catheter due to injuries (i.e., spinal cord injury, severe head trauma), and loss to follow up. The datasets used and/or analyzed during the current study are available from the corresponding author on request.

\section{Treatment groups}

Two treatment groups were developed (Figure 1). All patients presenting with PFUI to respective participating sites will be entered in the study. Prior to study initiation, centers must designate which treatment group they will join, which may be the closest to their current practice or if there is no preference will be assigned by the primary investigator to balance the number of centers in each group. If patients are treated outside of the treatment group for a given center, they will be excluded from the study. Each patient will undergo an attempt at simple retrograde catheter placement and if this fails the patient will undergo cystoscopy and a gentle attempt to traverse the gap and place a catheter retrograde with the scope. Usually this would be accomplished by passing the scope past the defect into the bladder and leaving a wire in the bladder exiting the meatus and penis. Then using a Seldinger technique, a catheter is placed over the wire, which serves as a guide, into the bladder. Among those failing retrograde catheter placement by attempting both methods, urologists will manage the injury with PR (endoscopic or open) (group 1) or SPT placement (group 2) as outlined in Figure 1.

Important aspects of these treatment groups are the 
Table 1 Outcome measures

\begin{tabular}{|c|c|}
\hline Outcome measures & Data point/data collection tool \\
\hline \multicolumn{2}{|l|}{ Primary outcome } \\
\hline Urethral obstruction & $\begin{array}{l}\text { Atraumatic passage of a } 17-\mathrm{F} \text { flexible } \\
\text { cystoscope }\end{array}$ \\
\hline \multicolumn{2}{|l|}{ Secondary outcomes } \\
\hline \multirow{6}{*}{$\begin{array}{l}\text { Treatment rates for } \\
\text { urethral obstruction }\end{array}$} & Dilation \\
\hline & DVIU \\
\hline & Urethroplasty \\
\hline & $\mathrm{ClC}$ \\
\hline & Indwelling catheter \\
\hline & Urinary diversion \\
\hline \multirow{8}{*}{$\begin{array}{l}\text { Urethroplasty } \\
\text { complexity }\end{array}$} & Gap length \\
\hline & Bulbar mobilization length \\
\hline & Corporal splitting \\
\hline & Inferior/total pubectomy \\
\hline & Corporal rerouting \\
\hline & Total obstruction \\
\hline & Diverticulum \\
\hline & Fistula \\
\hline \multirow[t]{4}{*}{ Erectile dysfunction } & Sexual Health Inventory for Men (SHIM) \\
\hline & ED intervention \\
\hline & (I) Pharmacologic \\
\hline & (II) Surgical \\
\hline \multirow[t]{5}{*}{ Incontinence rate } & Procedures for incontinence \\
\hline & (I) Bulking injections \\
\hline & (II) Artificial urinary sphincter \\
\hline & (III) Urethral sling \\
\hline & (IV) Other incontinence procedures \\
\hline $\begin{array}{l}\text { Post-injury } \\
\text { complications }\end{array}$ & Clavien-Dindo grading \\
\hline
\end{tabular}

DVIU, direct vision internal urethrotomy; CIC, clean intermittent catheterization; ED, erectile dysfunction.

need to perform retrograde cystoscopy exam and attempted retrograde catheter placement, regardless of whether patients are being treated with PR or SPT placement alone. The purpose of this is to eliminate selection bias and create homogenous comparator groups by determining if patients have partial or minor urethral injuries traversed easily with a retrograde cystoscope. Demographics will be collected including age, BMI, comorbidities (diabetes, stroke, peripheral vascular disease, myocardial infarction within 6 months, congestive heart, chronic obstructive pulmonary disease, end stage renal disease, failure, cirrhosis, tobacco use, alcohol use, illicit drug use). Additional index injury characteristics will include overall injury severity score (17), concomitant injuries, method of urethral injury diagnosis (urethrogram, cystogram, or cystoscopy), injury management, and trauma/urinary complications quantified by the Clavien-Dindo grading system (18).

Sexual function will be followed with the Sexual Health Inventory for Men (SHIM) (19). Potential delayed complications including urinary tract infection, fistula, urethral diverticulum will also be recorded.

\section{Study aims}

Aim 1: determine if there is a significant reduction in urethral obstruction based upon PR.

Aim 2: determine if urethroplasty complexity is reduced by PR.

Aim 3: evaluated the effect of $\mathrm{PR}$ on various clinical outcomes and complications associated with PFUI.

\section{Outcomes}

Primary and secondary outcomes are summarized in Table 1 .

\section{Primary outcome}

\section{Urethral obstruction}

Urethral obstruction will be determined by inability to atraumatically pass a flexible cystoscope retrograde through the urethra. Prior studies have demonstrated the large heterogeneity in urethral stricture definitions and follow up practice patterns $(20,21)$. Direct exam of the urethra at the site of injury or previous urethroplasty is a very accurate way of determining anatomic occurrence of a stricture or obstruction (21). Functional success is not as well defined and can be based upon patient's symptoms or need for additional procedures. Given the large number participating centers, it is not practical to monitor compliance with the extensive questionnaires needed to define patient's symptoms and outcomes.

\section{Secondary outcomes}

\section{Urethral obstruction treatment}

Treatment rate of urethral obstruction after injury, 
including: urethroplasty, direct vision internal urethrotomy (DVIU), urethral dilation, intermittent self-dilation, chronic dependence upon Foley catheter or SPT due to obstruction, and other types of urinary diversion. The need for secondary procedures including urethroplasty is a very important outcome measurement for the study. For instance, patients may experience obstruction of the urethra as assessed in the primary outcome; however, they may be asymptomatic and not require urethroplasty. Alternatively, these men, although symptomatic, might respond well to less invasive management, such as simple endoscopic treatment (DVIU or dilation) and may not need urethroplasty.

\section{Urethroplasty complexity}

Urethroplasty complexity as measured by: the length of the urethral gap, degree of bulbar mobilization, need for: corporal splitting, inferior or total pubectomy, corporal rerouting, abdominal-perineal approach, and presence of urethral diverticulum, urethral fistula, or complete obstruction of the urethra at the time of urethroplasty (versus a patent but narrowed urethral lumen). Advocates of $\mathrm{PR}$ argue that even if the procedure does not have as much success as some studies report, urethroplasty is made much easier, because the severed ends of the urethra are brought into rough alignment and there may be less of a urethral gap or there may be a persistent stenotic lumen rather than complete obstruction (12). Posterior urethroplasty has been described as a step-wise approach with four steps, each used to increase distal bulbar mobility to reach the prostate apex. The four steps are (I) bulbar mobilization; (II) corporal splitting; (III) inferior pubectomy; and (IV) corporal rerouting. Urethroplasties have been described as more complex when more steps are required, however, assessing urethroplasty complexity is not as simple, as surgeons may use different techniques at different frequencies depending upon their training and experience with posterior urethroplasty. A good example of this is corporal rerouting, which is rarely utilized by surgeons trained at the San Francisco General Hospital and the mentees of Dr. Jack McAninch, but utilized in up to $35 \%$ of patients treated at Duke and would likely have a higher incidence in patients treated by surgeons trained by Dr. George Webster $(5,20)$. Some intraoperative variables, however, should be consistent between surgeons, such as the measurement of the urethral gap, persistent urethral lumen rather than complete obliteration, and the presence of fistula or diverticulum.

\section{Erectile dysfunction (ED)}

Rates of ED will be measured by the SHIM, use of medical and/or surgical management, and questions about the presence of ED (19). Studies have demonstrated moderate correlation between SHIM and patient ED self-assessment (22). There have been few comparisons of ED in the more recent series of PR utilizing endoscopic realignment $(11,16,23)$. The SHIM has been used for assessment of ED post operatively after urethroplasty and will be administered in follow-up and after urethral specific interventions, such as urethroplasty.

\section{Incontinence}

Similar to ED rates, incontinence has only been compared between small PFUI management series $(11,16,23,24)$. In this study, incontinence rates will primarily be determined by interventions for incontinence. These interventions include artificial urinary sphincter placement, male urethral sling placement, injection of urethral bulking agent, and bladder neck reconstruction. Due to the large number of participating sites and the heterogeneity of multi-center data collection with regard to pad weight or incontinence questionnaires, these measures will not be used as outcome measures.

\section{Sample size determination}

Ninety-six men (48 per treatment group) will be required to detect a $15 \%$ difference in stricture rates between groups [ $80 \%$ power, 0.05 significance level for a 2-sided z-test of proportions, assuming a $20 \%$ loss to follow up or death rate and an intracluster correlation coefficient (ICC) of 0.03]. Since each institution will perform the acute management protocol of choice, we hypothesize this will invariably cause treatment effect clustering. In order to account for this, our sample size estimate was adjusted with an ICC or "the proportion of variation in the outcome that can be explained by the variation between clusters". Specifically, our sample size estimates were multiplied by a design effect of: $1+(n-1)$ $\times$ ICC (where $\mathrm{n}$ is the cluster size, which we assumed to be 3 patients/center) (25).

Demographics, index trauma characteristics, and retrograde urethrogram and/or cystoscopy findings (complete versus partial urethral disruption) will be compared between groups. The outcomes of interest as described above will be compared using both univariate and multivariate Cox regression models, given our interest in time-to-event outcomes, which include: acute injury complications, rates of subsequent urethral stricture, number of subsequent urethral procedures, complexity of urethroplasty, ED, and incontinence. Kaplan-Meier plots 

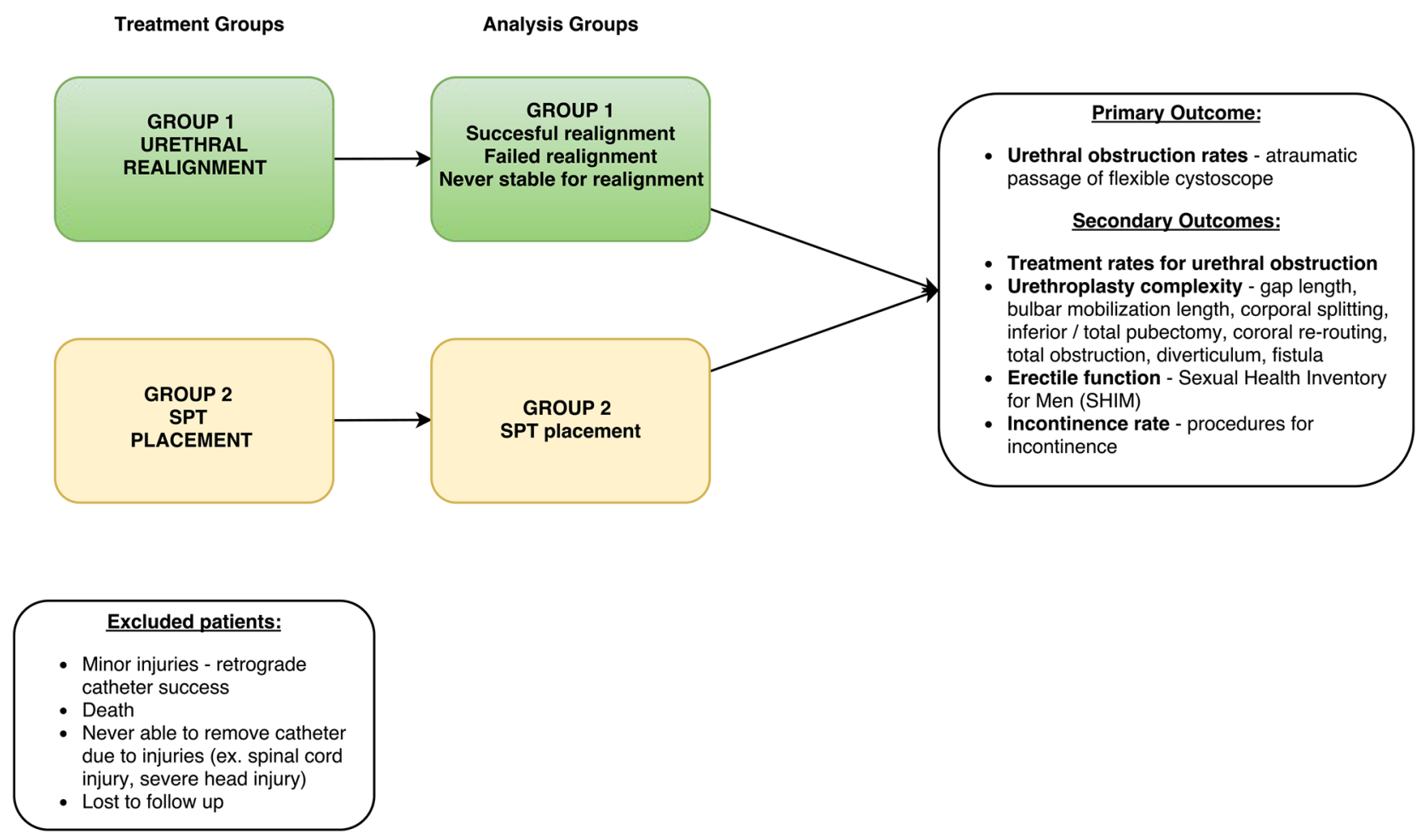

Figure 2 Analysis groups and outcome measures. SPT, suprapubic cystostomy tube.

will be used to visualize outcomes. Treatment and analysis groups are summarized in Figure 2.

\section{Trial duration}

Busy trauma centers manage/treat PFUI approximately $1-6 \times$ per year, thus our goal is to recruit at least 25 trauma centers and enroll patients for 3 years with a goal of 100 total patients with complete urethral disruption (50 per arm). Patients will be followed for the duration of the study or a minimum of a year. The trial duration will therefore be planned for 4 years.

\section{Safety}

All sites will obtain approval from their respective institutional review board (IRB). The data, which would be collected and de-identified would not pose any risk to the patients. A centralized de-identified database via REDCap will be used for data entry housed at University of Utah. REDCap allows protection of de-identified data by secure access based upon invitations to investigators and staff only.
In addition, participating institutions are made a 'designated access group' and can only view and modify their own data. Only the primary investigator and designated personnel can view all of the de-identified data.

\section{Results}

Data accrual began January $1^{\text {st }}$, 2016. Trauma centers have joined the study slowly over time. If centers followed a consistent group protocol than they entered data retrospectively back to January $1^{\text {st }} 2016$. If they did not follow a consistent method than once their IRB was approved they began following the designated group protocol.

\section{Discussion}

Acute management of PFUI remains a controversial topic primarily due to the lack of high quality evidence. While urinary diversion with SPT is an established initial PFUI management, numerous retrospective studies over the last 3 decades have demonstrated potential benefit of PR by reducing need for and or complexity of subsequent 
urethroplasty. No prospective study to date has evaluated the potential of $\mathrm{PR}$ in preventing urethral obstruction or the need for urethroplasty as compared to initial SPT diversion.

The major theory supporting urethral realignment is the ability of catheter placement to allow the torn ends of the urethra to heal together without a stricture (9). Retrospective series have demonstrated that PR may decrease the need for subsequent urethroplasty $(12-14,26)$. Notably, Mouraviev et al., in one of the largest studies of 91 PFUI patients found a stricture rate in only $49 \%$ of the PR group as compared to $100 \%$ in the SPT diversion group (12). This finding was corroborated by a recent meta-analysis by Barrett et al. evaluating nine studies, which also showed a decreased stricture rate among those undergoing PR compared to SPT placement alone (13). In this meta-analysis, the reduction was significantly lower in the PR group [odds ratio (OR) $=0.12,95 \%$ CI: $0.04-0.41$, $\mathrm{P}<0.001]$.

In contrast to these studies, a more recent contemporary series of 19 patients from Harborview, Seattle, Washington (a high-volume trauma and reconstructive urology center) reported that patients undergoing $\mathrm{PR}$, nearly universally required urethroplasty when they had a full urethral transection (23).

Furthermore, PR is not without risk in the acute injury period. PR, either endoscopically or open, can be difficult and may increase complications associated with the urethral injury and is best suited for the stable trauma patient (4). Often, posterior urethral distraction injuries may be associated with a pelvic hematoma. In a complete distraction injury, urethral instrumentation may lead to hematoma infection due to foreign body exposure, likely colonize shortly after placement (4). In addition to the immediate concerns, some studies have suggested that in the trauma population, the complication rate for urethroplasty in patients who have undergone prior urethral instrumentation might be higher, arguing against PR $(3,16,24)$. Early studies also suggested that ED and incontinence were higher in men undergoing PR. More recent studies suggest these complications are likely related to the underlying traumatic injury, rather than the choice of immediate urethral injury management (27) however, this has yet to be established in any longitudinal comparison study as proposed here.

Implicit in outcomes research is the need for longitudinal data bases that not only capture disease/ injury incidence, prevalence, and management but also follow post intervention functional status, patient reported health status, quality of life, and resource utilization (28). Although inferior to a prospective randomized controlled study, numerous data base projects have demonstrated the ability to pool data and produce meaningful prospective, observational research (29). Due to the relatively sparse incidence of PFUI, one could describe this as a rare event as it affects a small percentage of the US population (30). As such, many barriers to data collection have prevented randomized controlled trials of PFUI management. As we propose in this study, multi-institutional prospective cohort data bases have been suggested as a means to increase study power in rare disease processes (30).

The current proposed, prospective multi-institutional study would determine the utility of acute urethral realignment after PFUI and would increase the management level of evidence from $3 \mathrm{~A}$ (systematic review of case-control studies) to 2B (individual cohort study) (15).

\section{Acknowledgements}

None.

\section{Footnote}

Conflicts of Interest: This study is registered on clinicaltrials. gov (identifier NCT03195179).

Ethical Statement: The trial is in recruitment phase, all sites will obtain approval from their respective institutional review board (IRB). A waiver of consent for the study was granted by participating institutional review boards.

\section{References}

1. Bjurlin MA, Fantus RJ, Mellett MM, et al. Genitourinary injuries in pelvic fracture morbidity and mortality using the National Trauma Data Bank. J Trauma 2009;67:1033-9.

2. Koraitim MM. Pelvic fracture urethral injuries: evaluation of various methods of management. J Urol 1996;156:1288-91.

3. McAninch JW. Traumatic injuries to the urethra. J Trauma 1981;21:291-7.

4. Cooperberg MR, McAninch JW, Alsikafi NF, et al. Urethral reconstruction for traumatic posterior urethral 
disruption: outcomes of a 25-year experience. J Urol 2007;178:2006-10; discussion 2010.

5. Flynn BJ, Delvecchio FC, Webster GD. Perineal repair of pelvic fracture urethral distraction defects: experience in 120 patients during the last 10 years. J Urol 2003;170:1877-80.

6. Husmann DA, Wilson WT, Boone TB, et al. Prostatomembranous urethral disruptions: management by suprapubic cystostomy and delayed urethroplasty. J Urol 1990;144:76-8.

7. Richter ER, Morey AF. Urethral trauma. In: Wessells HB, McAninch JW. editors. Urological Emergencies: A Practical Guide. Totowa, NJ: Humana Press, 2005:57-69.

8. Webster GD, Mathes GL, Selli C.

Prostatomembranous urethral injuries: a review of the literature and a rational approach to their management. J Urol 1983;130:898-902.

9. Ormond JK, Cothran RM. Simple Method of Treating Complete Severance of Urethra Complicating Fracture of Pelvis. JAMA 1934;102:2180-1.

10. Patterson DE, Barrett DM, Myers RP, et al, Primary realignment of posterior urethral injuries. J Urol 1983;129:513-6.

11. Kim FJ, Pompeo A, Sehrt D, et al. Early effectiveness of endoscopic posterior urethra primary alignment. J Trauma Acute Care Surg 2013;75:189-94.

12. Mouraviev VB, Coburn M, Santucci RA. The treatment of posterior urethral disruption associated with pelvic fractures: comparative experience of early realignment versus delayed urethroplasty. J Urol 2005;173:873-6.

13. Barrett K, Braga LH, Farrokhyar F, et al. Primary realignment vs suprapubic cystostomy for the management of pelvic fracture-associated urethral injuries: a systematic review and meta-analysis. Urology 2014;83:924-9.

14. Moudouni SM, Patard JJ, Manunta A, et al. Early endoscopic realignment of post-traumatic posterior urethral disruption. Urology 2001;57:628-32.

15. Howick J, Chalmers I, Glasziou, P, et al. The Oxford Levels of Evidence 2. 2011. Oxford Centre for EvidenceBased Medicine. Available online: http://www.cebm.net/ index.aspx?o=5653

16. Tausch TJ, Morey AF, Scott JF, et al. Unintended negative consequences of primary endoscopic realignment for men with pelvic fracture urethral injuries. J Urol 2014;192:1720-4.
17. Baker SP, O'Neill B, Haddon W Jr, et al. The injury severity score: a method for describing patients with multiple injuries and evaluating emergency care. J Trauma 1974;14:187-96.

18. Dindo D, Demartines N, Clavien PA. Classification of surgical complications: a new proposal with evaluation in a cohort of 6336 patients and results of a survey. Ann Surg 2004;24:240-13.

19. Cappelleri JC, Rosen RC. The Sexual Health Inventory for Men (SHIM): a 5-year review of research and clinical experience. Int J Impot Res 2005;17:307-19.

20. Bullock TL, Brandes SB. Adult anterior urethral strictures: a national practice patterns survey of board certified urologists in the United States. J Urol 2007;177:685-90.

21. Erickson BA, Elliott SP, Voelzke BB, et al. Multiinstitutional 1-year bulbar urethroplasty outcomes using a standardized prospective cystoscopic follow-up protocol. Urology 2014;84:213-6.

22. Cappelleri JC, Siegel RL, Glasser DB, et al. Relationship between patient self-assessment of erectile dysfunction and the sexual health inventory for men. Clin Ther 2001;23:1707-19.

23. Leddy LS, Vanni AJ, Wessells H, et al. Outcomes of endoscopic realignment of pelvic fracture associated urethral injuries at a level 1 trauma center. J Urol 2012;188:174-8.

24. Culty T, Boccon-Gibod L. Anastomotic urethroplasty for posttraumatic urethral stricture: previous urethral manipulation has a negative impact on the final outcome. J Urol 2007;177:1374-7.

25. Donner A, Birkett N, Buck C. Randomization by cluster. Sample size requirements and analysis. Am J Epidemiol 1981;114:906-14.

26. Johnsen NV, Dmochowski RR, Mock S, et al. Primary Endoscopic Realignment of Urethral Disruption Injuries-A Double-Edged Sword? J Urol 2015;194:1022-6.

27. Aşci R, Sarikaya S, Büyükalpelli R, et al. Voiding and sexual dysfunctions after pelvic fracture urethral injuries treated with either initial cystostomy and delayed urethroplasty or immediate primary urethral realignment. Scand J Urol Nephrol 1999;33:228-33.

28. Pryor DB, Califf RM, Harrell FE Jr, et al. Clinical data bases. Accomplishments and unrealized potential. Med Care 1985;23:623-47.

29. Lubeck DP, Litwin MS, Henning JM, et al. The CaPSURE database: a methodology for clinical practice 
and research in prostate cancer. CaPSURE Research Panel. Cancer of the Prostate Strategic Urologic Research Endeavor. Urology 1996;48:773-7.

Cite this article as: Moses RA, Selph JP, Voelzke BB, Piotrowski J, Eswara JR, Erickson BA, Gupta S, Dmochowski RR, Johnsen NV, Shridharani A, Blaschko SD, Elliott SP, Schwartz I, Harris CR, Borawski K, Figler BD, Osterberg EC 3rd, Burks FN, Bihrle W 3rd, Miller B, Santucci RA, Breyer BN, Flynn B, Higuchi T, Kim FJ, Broghammer JA, Presson AP, Myers JB; from the Trauma and Urologic Reconstruction Network of Surgeons (TURNS). An American Association for the Surgery of Trauma (AAST) prospective multi-center research protocol: outcomes of urethral realignment versus suprapubic cystostomy after pelvic fracture urethral injury. Transl Androl Urol 2018;7(4):512-520. doi: 10.21037/ tau.2017.11.07
30. Gagne JJ, Thompson L, O'Keefe K, et al. Innovative research methods for studying treatments for rare diseases: methodological review. BMJ 2014;349:g6802. 